\title{
Brown Adipose Tissue: A New Target for Antiobesity Therapy
}

\author{
Anna Meiliana ${ }^{1,2 *}$ and Andi Wijaya ${ }^{1,2 *}$ \\ ${ }^{1}$ Post Graduate Program in Clinical Biochemistry, Hasanuddin University, Makassar \\ ${ }^{2}$ Prodia Clinical Laboratory, Jakarta \\ *Address correspondence to this author at: Prodia Clinical Laboratory, JI. Cisangkuy No.2, Bandung. \\ E-mail: anna_m@prodia.co.id, andi_w@prodia.co.id
}

\section{Abstract}

$\mathrm{B}$

ACKGROUND: Human fat consist of white and brown adipose tissue (WAT and BAT). Though most fat is energy - storing WAT, the thermogenic capacity of even small amounts of BAT makes it an attractive therapeutic target for inducing weight loss through energy expenditure.

CONTENT: Over the past year, several independent research teams used a combination of positron - emission tomography and computed tomography (PET/CT) imaging, immunohistochemistry, and gene and protein expression assays to prove conclusively that adult humans have functional BAT. BAT is important for thermogenesis and energy balance in small mammals, and its induction in mice promotes energy expenditure, reduces adiposity, and protects mice from diet - induced obesity. The thermogenic capacity of BAT is impressive. In humans, it has been estimated that as little as $50 \mathrm{~g}$ of BAT could utilize up to $20 \%$ of basal caloric needs if maximally stimulated.

SUMMARY: The obesity pandemic requires new and novel treatments. The past few years have witnessed multiple studies conclusively showing that adult humans have functional BAT, a tissue that has a tremendous capacity for obesity - reducing thermogenesis. Novel therapies targeting BAT thermogenesis may be available in the near future as therapeutic options for obesity and diabetes. Thermogenic ingredients may be considered as functional agents that could help in preventing a positive energy balance and obesity.
KEYWORDS: Brown adipose tissue, thermogenesis, energy expenditure, antiobesity therapy.

\section{Introduction}

Obesity has reached epidemic proportions, and complications related to obesity contribute substantially to health care costs and mortality. Since the accumulation of fat is the net result of a prolonged state of imbalance between energy intake and energy expenditure, one would think that an ideal fat mass in obese persons could be achieved relatively simply by either decreasing food intake or increasing energy expenditure, ultimately causing a sustained negative energy balance. Unfortunately, this is not so easy to achieve, because evolutionary pressure has rewarded those individuals and species able to store sufficient energy to survive famines; also, the unrestricted availability of food represents an unnatural condition (1). Currently, most interventions, whether behavioral or pharmacologic, are aimed at the energy - intake side of the equation and result in only moderate, often temporary improvements, with the notable exception of bariatric surgery.

Interventions designed to increase energy expenditure are relatively limited. An increase in physical activity, although effective, is not easy to sustain. The pharmacologic approach has also been disappointing. Supraphysiologic doses of thyroid hormones or adrenergic agonists result in an increase in energy expenditure, but their systemic adverse events preclude their use for the treatment of obesity (2). 
The development of obesity not only depends on the balance between food intake and caloric utilization but also on the balance between white adipose tissue, which is the primary site of energy storage, and brown adipose tissue, which is specialized for energy expenditure (3).

Brown adipose tissue represents a natural target for the modulation of energy expenditure. This tissue is far from being a fat depot. When activated, it requires the uptake of substrate from the circulation, mostly free fatty acids, but also glucose. The physiologic role of brown adipose tissue in small mammals (and human newborns) is the maintenance of core temperature. In brown adipose tissue, mitochondria release chemical energy in the form of heat by means of the uncoupling of the oxidative phosphorylation, making the process of respiration exceedingly inefficient. This phenomenon is mediated by the uncoupling protein 1 (UCP1), which renders the inner membrane of the mitochondria "leaky" and hence releases energy in the form of heat rather than storing it as ATP (4). UCP1 is, in turn, regulated by triiodothyronine, which is generated within brown adipose tissue by means of 5' deiodination of the prohormone thyroxine, effectively creating a local, tissue-specific hyperthyroid state in the absence of changes in circulating levels of thyroid hormones (5). $\beta 3$-adrenergic signaling plays an important role in the modulation of this process, and recent evidence indicates that food intake results in a similar activation (6), suggesting that brown adipose tissue could play an important role in short-term energy homeostasis.

The findings in support of functional $\mathrm{hBAT}$ are highly relevant to both basic research and clinical medicine, since they offer the possibility of regulating human energy expenditure. Increased energy expenditure would be very valuable as a therapeutic approach to obesity and obesity-related diseases such as type 2 diabetes. This could involve recruitment of brown adipocyte progenitors and subsequent differentiation as one way of enhancing the hBAT compartments (7).

\section{Rediscovery of BAT in Adult Humans}

During 2009, several studies led to a paradigm shift in our understanding of the potential role of BAT in adult humans. Although it has been known that adult humans may have small pockets of BAT at postmortem examination (8), attempts to find functional BAT during life (9) or utilize its thermogenesis for weight loss $(10,11)$ have been largerly unsuccessful $(12,13)$, so BAT was thought to be biologically irrelevant in adult humans. Despite this view in the endocrine community, reports in the radiological literature using positron - emission tomography and computed tomography (PET/CT) began to suggest a different point of view, PET, or positron emission tomography, uses radiotracers such as ${ }^{18} \mathrm{~F}$-fluorodeoxyglucose $\left({ }^{18} \mathrm{~F}\right.$-FDG) to measure the metabolic activity of tissues. CT provides high - resolution anatomical detail. Fusion of the PET and CT images provides both functional and structural information in a single image. In the course of using PET/CT to detect and stage tumors (14) radiologists noted small, but distinct, nontumor collections of adipose tissue, that is, fat with high uptake of ${ }^{18} \mathrm{~F}$-FDG $(15,16)$. Although this tissue was often called "BAT" in the radiological literature, internists, and endocrinologists doubted these findings and persisted with the view that adult humans have no functional BAT.

Using an experimental approach, van Marken Lichtenbelt and colleagues (17) examined the presence, distribution, and activity of brown adipose tissue and its potential role in energy metabolism in a group of volunteers. The study used ${ }^{18} \mathrm{~F}$-FDG PET-CT to assess the cold-stimulated brown-adipose-tissue activity in relation to metabolic measures, including energy expenditure at rest and core and surface temperatures. All the subjects, with the notable exception of the most obese volunteer, displayed functional brown adipose tissue; the activity of the tissue correlated inversely with BMI and the percentage of body fat and correlated directly with energy expenditure during rest. A substantial increase in energy expenditure was observed in all the subjects exposed to mild cold, but the authors found no correlation between cold-induced thermogenesis and brown-adipose-tissue activity (12).

The common message from these studies is that brown adipose tissue is present and active in adult humans, and its presence and activity are inversely associated with adiposity and indexes of the metabolic syndrome $(2,18)$. Taken together, these studies point to a potential "natural" intervention to stimulate energy expenditure: turn down the heat and burn calories (and reduce the carbon footprint in the process). Obviously, this strategy is an oversimplification, and one should expect compensatory mechanisms aimed at maintaining the energy homeostasis - that is, an increase in energy intake in response to a loss of energy secondary to cold exposure. Nonetheless, these studies, by showing the presence and activity of brown adipose tissue in adult humans, are a powerful proof of concept that this tissue might be used as a target for interventions, pharmacologic and environmental, aimed at modulating energy expenditure (2). 


\section{BAT Function and Biology}

Cold weather and scarcity of food have accompanied us and constituted serious threats to our survival for the entire duration of our species' history. Fortunately, two tissues have been selected by nature to help us deal with these difficulties: WAT and BAT. While WAT helps us compensate for fluctuating availability of energy, BAT keeps us warmat least during our first days of extrauterine life (19).

Brown and white adipocytes have widely different morphologies, mainly in terms of the shape of the lipid droplets and of mitochondria. Lipids are organized as multiple, small droplets in brown adipocytes and as a single, large lipid droplet in white adipocytes. Mitochondria are large and numerous and are endowed with laminar cristae in brown adipocytes, whereas they are small and elongated, with randomly oriented cristae, in white adipocytes. These differences correspond to different functional roles- respectively, heat production with energy dissipation and energy storage and distribution. The function of brown adipocytes is critically related to UCP1, the mitochondrial protein uniquely expressed in this cell type and therefore considered as the molecular marker of BAT (20). Despite their different anatomy and functional roles, brown and white adipocytes are found together in dissectable structures, the fat depots (21).

The recent demonstration of considerable amounts of metabolically active brown BAT in many adult humans has renewed the scientific interest in the subject worldwide, mainly because of its antiobesity effects, documented in murine models. Genetic ablation of BAT or of all $\beta$-adrenergic receptors, which are responsible for its activation, induces obesity in mice (22), whereas ectopic expression of $\mathrm{UCP} 1$ - the protein-activating brown adipocyte thermogenesis uniquely found in BAT - in WAT results in resistance to obesity (23). Accordingly, administration of drugs capable of increasing BAT activity curbs obesity and related disorders, such as type 2 diabetes (21).

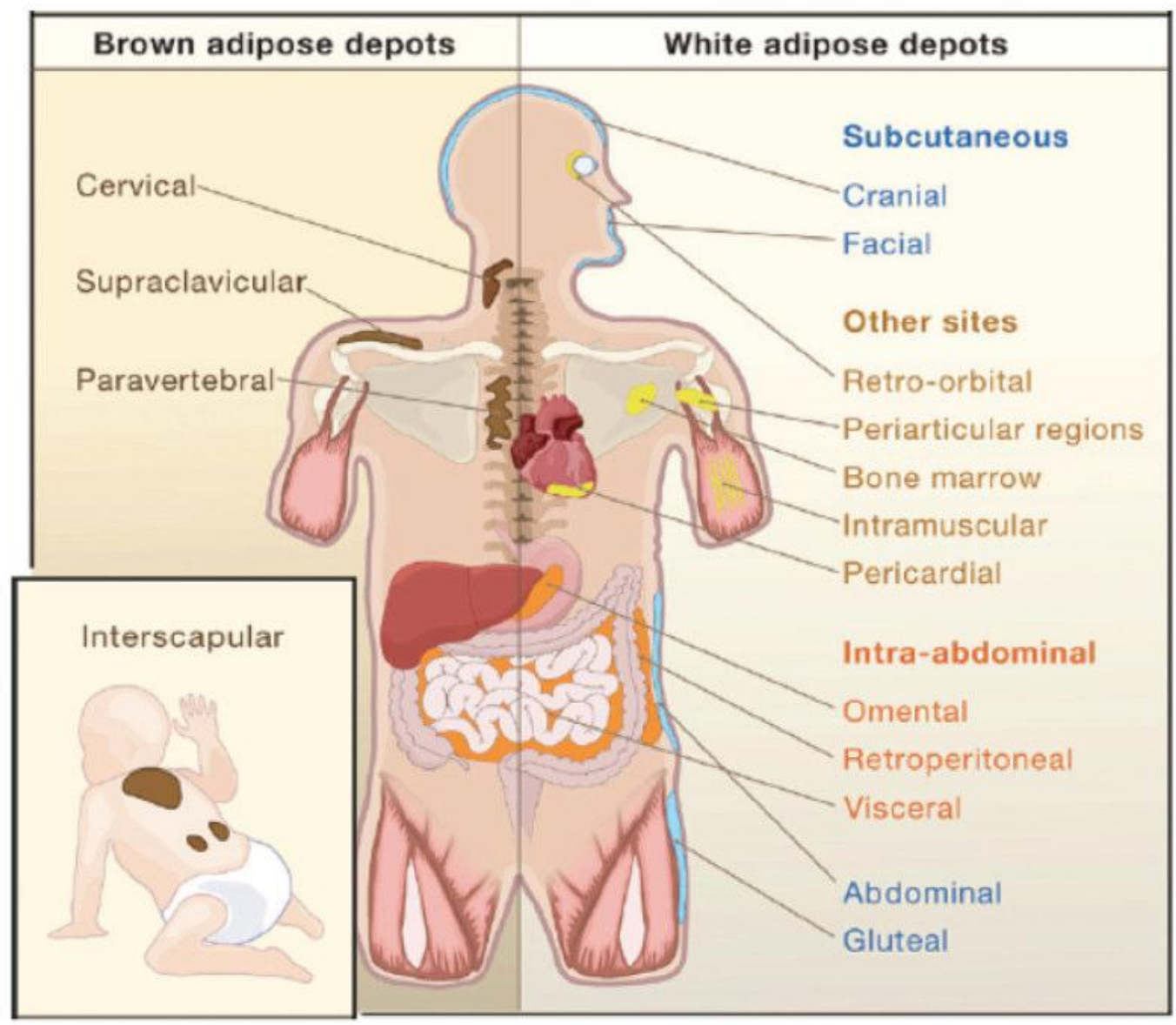

Figure 1. In humans, depots of WAT are found in areas all over the body, with subcutaneous and intra - abdominal depots representing the main compartments for fat storage. BAT is abundant at birth and still present in adulthood but to a lesser extent (Adapted with permission from Elsevier Inc.) (3). 
The function of BAT is to transfer energy from food into heat; physiologically, both the heat produced and the resulting decrease in metabolic efficiency can be of significance. Both the acute activity of the tissue, i.e., the heat production, and the recruitment process in the tissue (that results in a higher thermogenic capacity) are under the control of norepinephrine released from sympathetic nerves. In thermoregulatory thermogenesis, BAT is essential for classical nonshivering thermogenesis (this phenomenon does not exist in the absence of functional BAT), as well as for the cold acclimation-recruited norepinephrine-induced thermogenesis. Heat production from BAT is activated whenever the organism is in need of extra heat, e.g., postnatally, during entry into a febrile state, and during arousal from hibernation, and the rate of thermogenesis is centrally controlled via a pathway initiated in the hypothalamus. Feeding as such also results in activation of BAT; a series of diets, apparently all characterized by being low in protein, result in a leptin-dependent recruitment of the tissue; this metaboloregulatory thermogenesis is also under hypothalamic control. When the tissue is active, high amounts of lipids and glucose are combusted in the tissue. The development of BAT with its characteristic protein, $\mathrm{UCP} 1$, was probably determinative for the evolutionary success of mammals, as its thermogenesis enhances neonatal survival and allows for active life even in cold surroundings (20).
Among the factors that influence the brown adipocyte, norepinephrine is both the most important and the most wellstudied. This effector is most significant physiologically, not only for the acute thermogenic process but also for the control of cell proliferation, advanced cell differentiation, and apoptosis.

In mature brown adipocytes, norepinephrine interacts with all three types of adrenergic receptors: $\beta 1, \alpha 2$, and $\alpha 1$; these receptor types are associated with activation of different signaling pathways in the brown adipocytes, as will be detailed below. The most significant and the most studied pathway is the pathway for $\beta$-adrenergic stimulation of thermogenesis.

Of the three subtypes of $\beta$-adrenergic receptors, the $\beta 3$-adrenoceptor is the most significant in mature brown adipocytes from rodents. $\beta 1$-Adrenoceptors are also expressed in mature brown adipocytes, but they are not coupled to any significant extent to signaling processes in these cells; they are, however, coupled to cAMP production in brown preadipocytes, which means that in membrane preparations from total BAT, both receptor subtypes will be functional. $\beta 2$-Adrenoceptors are not expressed in the brown adipocytes themselves, but they are expressed in the tissue and can be observed as binding sites in membrane preparations from BAT. These $\beta 2$-adrenoceptors are probably predominantly localized to the vascular system (20).

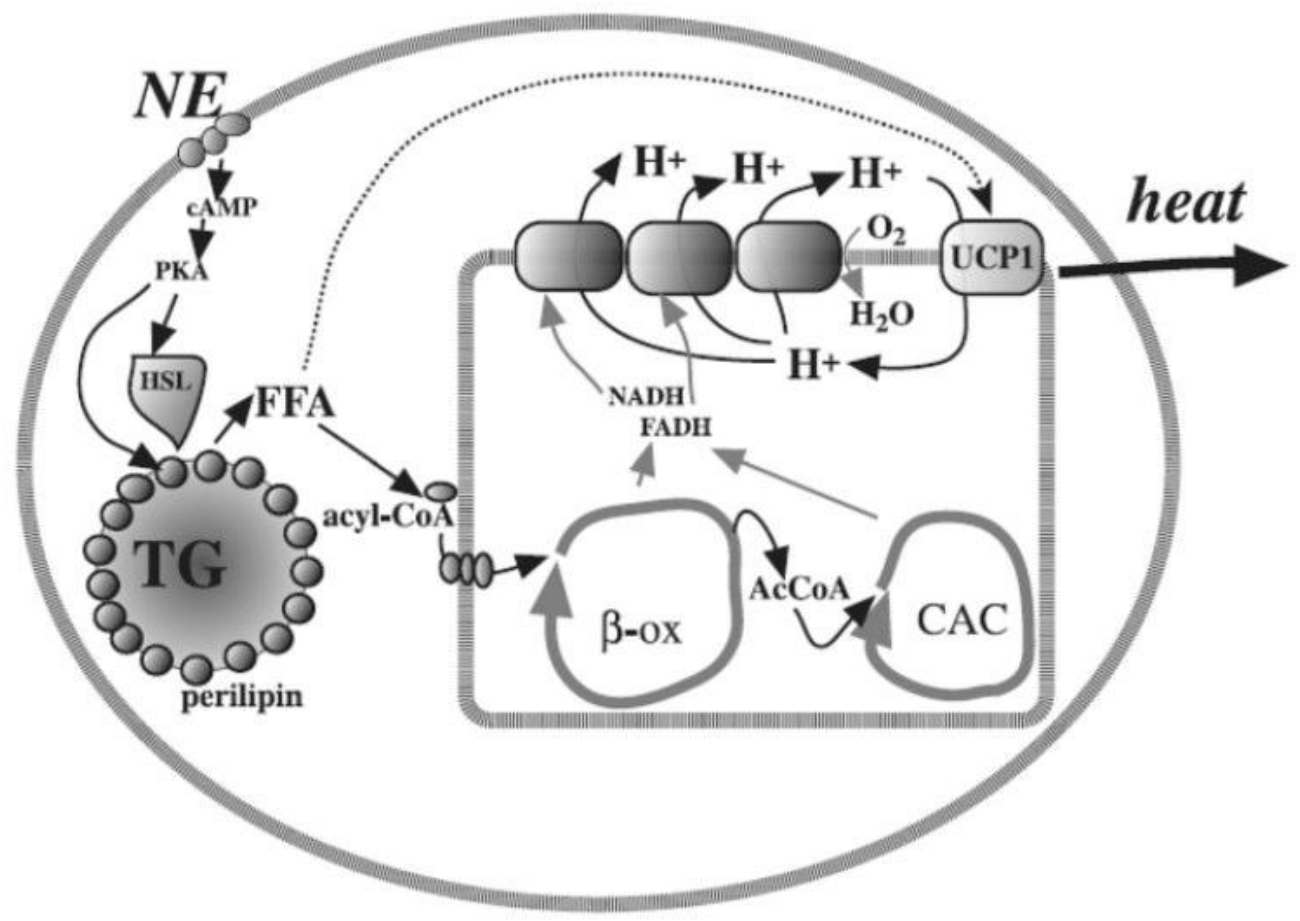

Figure 2. Norepinephrine induced stimulation of thermogenesis in brown adiposytes: events downstream of the protein kinase $A$ (PKA) activation (adapted with permission from The American Physiological Society) (20). 
Free fatty acids (FFA) activated to acyl CoAs by acyl-CoA synthetase are first transferred to acyl-carnitine by the highly expressed muscle form of carnitine palmitoyltransferase I (M-CPT I), which is the CPT I form found in both brown and WAT and which is very sensitive to inhibition by malonyl CoA. The acyl-carnitine probably enters the mitochondria through the carnitine transporter (not as yet explicitly described in BAT) and is probably reconverted to acyl CoA by CPT II. The ensuing $\beta$-oxidation ( $\beta$-ox) of the fatty acids (acyl CoAs) as well as the activity of the citric acid cycle (CAC) lead to the formation of the reduced electron carriers FADH and NADH, which are then oxidized by the electron transport chain (respiratory chain) ultimately through oxygen consumption. This results in a pumping out of protons from the mitochondria and the formation of a proton-motive force that drives the protons back into the mitochondrial matrix through the uncoupling protein UCP1. The energy stored in the proton-motive force is then released as heat (20).

What is a matter for debate is whether BAT thermogenesis burns off excess calories in a state of positive energy balance to maintain energy homeostasis as a major physiological function. This concept emerged from experiments in the 1970s when Rothwell and Stock observed that rats fed a cafeteria diet (composed of junk foods high in fats and sugars) gained less weight than expected from caloric intake, and they proposed that excess unaccounted calories were being burned off by the induction of BAT thermogenesis. Accordingly, BAT thermogenesis was proposed as a mechanism not only for protecting body temperature, but also to protect against obesity and the development of insulin resistance (24).

We are now entering another heady era for research that seeks to relate directly the function of BAT thermogenesis to the regulation of body weight. The basis of this renewed interest is the realization that PET/CT images of active glucose uptake in humans are sites of active BAT. The function of BAT as a thermogenic mechanism for the regulation of body temperature is well established and indisputable. In the key experiment, Foster and Frydman showed that following norepinephrine administration to cold-acclimated rats, blood flow through BAT accounted for $33 \%$ of cardiac output even though BAT mass constitutes only $1.3 \%$ of total body mass. In the same animals, skeletal muscle constituted $38 \%$ of body mass, yet accounted for only $8.4 \%$ of cardiac output. The effects of this thermogenic system for maintaining body temperature on energy balance are correspondingly impressive (25).

Expenditure of energy to balance food intake would come principally from physical activity and maintenance of body temperature in individuals in harmony with their environment; this would exclude most modern humans. The realization this past year that significant levels of BAT continue to exist in adult humans has opened the door to research that will aim to determine how this remarkable thermogenic system may be associated with the obesity epidemic and to discover new ways to utilize the potential to expand and activate BAT thermogenesis to prevent or reduce obesity in individuals (24).

\section{Thermoregulatory Thermogenesis and Metaboloregulatory Thermogenensis}

Two main physiological purposes of brown fat-derived thermogenesis can be identified. One is what can be understood physiologically as thermoregulatory thermogenesis, the function of which is to produce heat for defense of regulated body temperature. The second is a "metabolic inefficiency thermogenesis," metaboloregulatory thermogenesis, the function of which seems to be to allow for combustion of excess energy intake, perhaps with the purpose of allowing an "extraction" of essential food constituents (notably protein).

When an animal is acutely exposed to a "low" environmentaltemperature,itneedsextraheattocompensate for the increased heat loss, to defend its body temperature. Principally, "low" here means any temperature lower than that referred to as "the lower critical temperature of the thermo-neutral zone." For most experimental animals (and for naked humans), this temperature lies close to $30^{\circ} \mathrm{C}$ (Fig 3 ). The extra heat needed to defend the body temperature becomes, however, smaller in relative magnitude the larger the animal becomes. There are two reasons for this: basal metabolism increases, for reasons still unknown, in proportion to the body weight to the power of 0.75 , i.e., it is $\sim 11 \mathrm{ml} \mathrm{O} 2 \cdot \mathrm{min}^{-1} \bullet \mathrm{kg}^{-0.75}$ irrespective of mammalian species studied, whereas the surface (i.e., the area from which heat is lost to the surroundings) increases, for geometrical reasons, only in proportion to body weight to the power of 0.67 . That the power functions are different means that the basal metabolism can provide more and more of the necessary heat the larger an animal becomes. The greater body weight also makes it possible for animals to carry relatively more insulation. The outcome is that under "normal" (room temperature) conditions, common experimental animals constantly maintain a metabolic rate about double their minimal metabolism, merely to defend their body temperature. Chronically, this exposure to normal room temperature will lead to the recruitment of a certain 
capacity for nonshivering thermogenesis. Larger animals, including humans, do not normally require this "extra" metabolism for thermal homeostasis and consequently have not recruited such a capacity. It is not impossible that this difference between large and small animals explains some difficulties in extrapolating from experimental animals to humans in these matters (20).

There is ample evidence that prolonged cold acclimation leads to BAT recruitment, as analyzed by any parameter. Notably, the total amount of UCP1 increases some 10 -fold or more due to cold acclimation, but also an increase in total cellularity, total amount of mitochondria, mitochondrial enzymes, fatty acid oxidizing enzymes, etc., contribute to the vastly enhanced oxidative capacity. That BAT is activated in the cold was most clearly demonstrated in the classical blood flow experiments of Foster and Frydman. Based on measurements of tissue blood flow distribution and the arterial-venous oxygen difference over the tissue, it was estimated by these authors that BAT could use at least some $60 \%$ of the extra oxygen used in cold-acclimated animals in the cold, i.e., BAT could be responsible for at least $60 \%$ of non-shivering thermogenesis. Through this, BAT was accepted as the major site for non-shivering thermogenesis (20).

Metaboloregulated thermogenesis has been interpreted as a form of defense against a caloric load, and also as a way of surviving on diets that are low in essential nutrients without there being any pronounced effects on energy depots, since excess energy will be dissipated by active BAT. Another possibility is that readily available calories will signal to increase the BAT compartment - not necessarily for the purpose of dissipating energy acutely, but rather to increase its size, since energy appears to be abundant, which would perhaps confer an enhanced capacity to adapt to sudden bursts of cold in the environment.

It has been estimated that $40-50 \mathrm{~g}$ of maximally stimulated BAT in man could correspond to as much as $20 \%$ of energy expenditure, equivalent to $20 \mathrm{~kg}$ of body weight, over a year. Thus, even a fraction of this effect could be highly relevant in terms of human energy expenditure. It should be pointed out that while in the mouse BAT makes up some $5 \%-10 \%$ of the total body weight, the corresponding value for infants has been estimated to be approximately $2 \%-5 \%$ and in adults about $0.05 \%-0.1 \%$ (19).

Two pathways for diet control of BAT recruitment status. First: the intake of a meal is signaled to the brain through blood-borne substances that interact with the centers in the brain that regulate BAT activity. Due to the repetitive (although intermittent) effect of single meals, a certain degree of BAT recruitment is maintained. Second: due to overfeeding (which may e.g., be secondary to

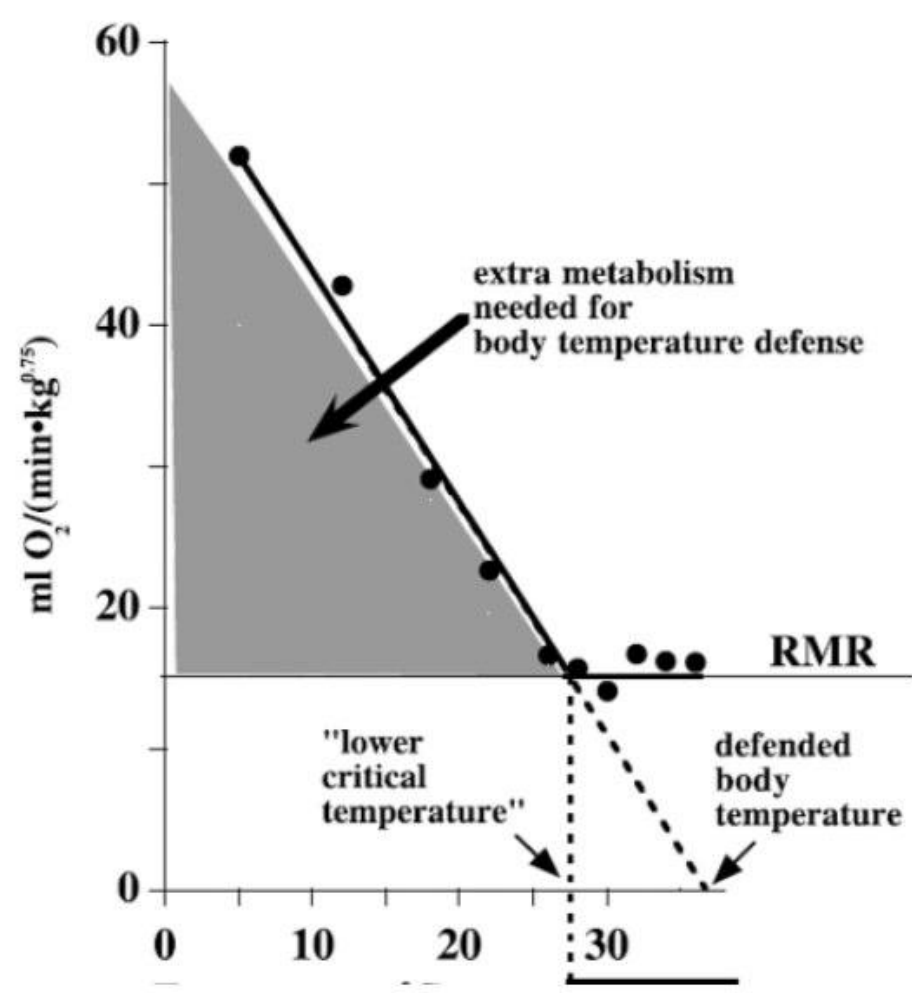

Figure 3. Thermoregulatory metabolic response to environmental temperature. Principal sketch is based on actual observations ( $\bullet$ ) on mice. RMR < resting metabolic rate; TNZ, thermoneutral zone (Adapted with permission from American Physiological Society) (20)

protein dilution in the diet, as occurs in cafeteria diets and in most high-fat diets), obesity will develop, leading to increased leptin signaling to the centers in the brain that regulate BAT activity. This will lead to a positive correlation between degree of obesity and recruitment state of BAT. Suggest that this is the basis for so-called "diet-induced thermogenesis" (which thus rather may be considered to be "obesity-induced thermogenesis"). The effect of this pathway is that obesity is reduced due to decreased metabolic efficiency. Obviously, failures in the leptin signaling pathway, such as are found in $o b / o b, d b /$ $d b$, and $f a / f a$ animals, will lead to augmented obesity as the increased food intake is not counteracted by increased BAT activity. Accordingly, different sensitivities of the leptin feedback system may be involved or even be explanatory for the differences between individuals (or between inbred mouse strains) in their responses to high-fat diets (20). 


\section{Transcriptional Control of BAT Development and Differentation}

Despite the differences in the developmental origins and physiological functions of brown and white adipocytes, both cell types share a very similar transcriptional cascade that controls the process of fat differentiation. Detailed studies of white fat differentiation had previously identified PPAR $\gamma$ (peroxisome proliferator-activated receptor-g) and the C/EBPs (CCAAT/enhancer-binding proteins) as key transcription factors driving fat cell differentiation (27). Indeed,PPAR $\gamma$ is absolutely necessary for both white fat and brown fat cell development. C/EBPs function cooperatively with PPAR $\gamma$ and promote a transcriptional cascade that promotes and maintains the stable differentiated state of adipocytes. Brown fat cell differentiation requires PPAR $\gamma$ but, importantly, this factor alone is not sufficient to drive mesenchymal cells into a brown fat program (27).

\section{Forkhead Box C2}

Forkhead box C2 (FOXC2) is a member of the forkhead/ winged helix transcription factor family whose gene expression is enriched in the adipose tissues of human and mouse. Enerback and colleagues reported that transgenic expression of FOXC2 in the adipose tissue induced the emergence of brown fat-like cells in WAT, with increased mitochondria and elevated expression of thermogenic genes, including UCP1 and PPAR $\gamma$-coactivator- $1 \alpha$ (PGC$1 \alpha)$ (28). Notably, transgenic expression of FOXC2 counteracts many obesity-associated pathologies, including insulin resistance and hypertriglyceridemia (28).

\section{PGC-1 $\alpha$ and Its Transcriptional Regulators}

PGC- $1 \alpha$ was originally identified from brown fat cells as a cold-inducible coactivator of PPAR $\gamma$ (29). Accumulating evidence indicates that PGC- $1 \alpha$ is a master regulator of mitochondrial biogenesis and oxidative metabolism in most cell types, including brown fat and skeletal muscle.

Several transcriptional regulators have been shown to control brown fat development and function, at least in part, through regulating the transcriptional activity or gene expression of PGC-1 $\alpha$. RIP140, a corepressor of many nuclear receptors, has been shown to suppress the transcriptional activity of PGC-1 $\alpha$ through a physical interaction. Genetic ablation of RIP140 causes the emergence of brown fat-like cells in WAT (30). Similarly, SRC2/TIF2/GRIP1, a member of the steroid receptor coactivator (SRC) family, represses PGC- $1 \alpha$ transcriptional activity. Loss of SRC2 function leads to an increase in adaptive thermogenesis and energy expenditure in vivo (31). $\mathrm{Rb}$ (retinoblastoma) protein and $\mathrm{p} 107$, another member of the $\mathrm{Rb}$ pocket protein family, also negatively regulate $\mathrm{PGC}$ $1 \alpha$ gene expression.Adipocytes derived from $\mathrm{pRb}$-deficient fibroblasts or embryonic stem cells exhibit a brown fat phenotype with high mitochondrial content and elevated expression of UCP1, PGC- $1 \alpha$, and mitochondrial genes (32). p107-deficient mice display a striking accumulation of brown fat-like cells in WAT, with multilocular lipid droplets, abundant mitochondria, and high levels of UCP1 and PGC-1a expression (33). The biological effect of p107 on brown fat development appears to be mediated through the repression of $\mathrm{pRb}$, because $\mathrm{pRb}$ levels are significantly reduced in Sca-1+ CD31- Lin- adipogenic precursors isolated from p107-deficient mice. Lastly, TWIST1, a helix-loop-helix-containing transcriptional regulator, has recently been reported as a negative regulator of PGC$1 \alpha$ function in brown fat. Genetic ablation or depletion of TWIST1 induces the expression of brown fat-selective genes, while over-expression of TWIST1 represses them in a PGC-1 $\alpha$-dependent fashion (34).

\section{PRDM16}

PRD1-BF-1-RIZ1 homologous domain containing protein-16 (PRDM16) is a $140 \mathrm{kDa}$ zinc finger protein that was originally identified at a chromosomal breakpoint of $\mathrm{t}(1 ; 3)(\mathrm{p} 36 ; \mathrm{q} 21)$-positive human acute myeloid leukemia cells (35). Study showed that PRDM16 expression was highly enriched in BAT compared with WAT (36). When ectopically expressed in white fat preadipocytes or myoblasts, PRDM16 induces a nearly complete brown fat genetic program. This includes mitochondrial biogenesis, increased cellular respiration, and expression of brown fat-selective genes, both the cAMP-inducible thermogenic genes (Ucp1, Pgc-1a, and Deiodinase-d2) and those BATselective genes that are not sensitive to cAMP (such as Cidea and Elovl3). 
Depletion of PRDM16 in primary brown fat cell precursors not only causes a near-total loss of brown fat characteristics but also, unexpectedly, causes the emergence of distinct morphological and genetic characteristics of skeletal myotubes in culture. These include the formation of syncytia and ectopic activation of skeletal musclespecific genes. Consistent with this, BAT from PRDM16deficient mice exhibits an abnormal morphology, with reduced expression of brown fat-selective marker genes and elevated expression of skeletal musclespecific genes (36). These results suggest that PRDM16 is a critical determinant of the brown fat lineage from myoblast progenitors during the embryonic development.

How does PRDM16 control the conversion of myoblastic precursors to brown fat? Recent studies demonstrated that PRDM16 forms a transcriptional complex with the active form of $\mathrm{C} / \mathrm{EBPb}$ (LAP) that is abundantly expressed both in brown fat and myoblasts (38). It has also been reported that $\mathrm{C} / \mathrm{EBPb}$ is a dominant transcription factor that controls cAMP-induced gene expression in brown fat cells (39). These studies indicate that a PRDM16-C/EBPb complex controls the initiating events of the conversion from myoblastic precursors to brown fat cells.

PRDM16 directly interacts with canonical transcription factors such as PPAR $\alpha, \operatorname{PPAR} \gamma$, and C/EBP family members and transcriptional coactivators PGC- $1 \alpha$ and PGC- $1 \beta$ through the two sets of zinc finger domains (ZF1 and ZF2). PRDM16 is also associated with the corepressors CtBP1 and 2 through its PLDLS motif. This interaction mediates the repressive action of PRDM16 on the expression of white fat cell-specific genes.

PRDM16-C/EBP $\beta$ transcriptional complex acts in Myf5-positive myoblastic precursors or preadipocytes to induce the expression of PPAR $\gamma$ and PGC-1 $\alpha$. PRDM16 coactivates PPAR $\gamma$ and PGC- $1 \alpha$, which then drives a brown fat differentiation program. The cAMP-dependent thermogenic gene program is potentiated by FOXC2 and PRDM16. RIP140, Rb/p107, and TWIST1 antagonize the expression or transcriptional activity of PGC- $1 \alpha$ and repress brown fat genetic program.

The signaling molecules that control the timing and specificity of PRDM16 expression and commitment to the brown adipose lineage are unknown. Bone morphogenic proteins (BMPs), members of the TGF- $\beta$ superfamily of secreted factors, have been suggested to facilitate adipogenic differentiation. Of particular interest is BMP7, which has been described as a selective and potent inducer of brown but not white adipogenesis in preadipocyte and multipotent fibroblast cultures (40). BMP7 treatment of fibroblast cultures is associated with induction of key brown adipogenic regulators such as PRDM16 and PGC$1 \alpha$, although the mechanisms that mediate this effect are not known. Importantly, BMP7-deficient embryos possess significantly reduced amounts of BAT that lack UCP1 expression (40).

Another member of the TGF- $\beta$ superfamily, GDF-3, has been shown to negatively regulate a thermogenic gene program in white fat depots; hence, this factor could also play a role in BAT development (41). Wnt activation represses both brown and white adipogenesis by suppressing the induction of PPAR $\gamma$ and $\mathrm{C} / \mathrm{EBP} \alpha$ in precursor cells (42). By contrast, Wnt ligands are crucial inductive cues for embryonic muscle formation through direct activation of muscle determination factors. Fibroblast growth factors (FGFs) -16,-19 (FGF15 in mouse), and -21 have also been implicated in the regulation of brown adipogenesis and thermogenic activity of brown fat (43).

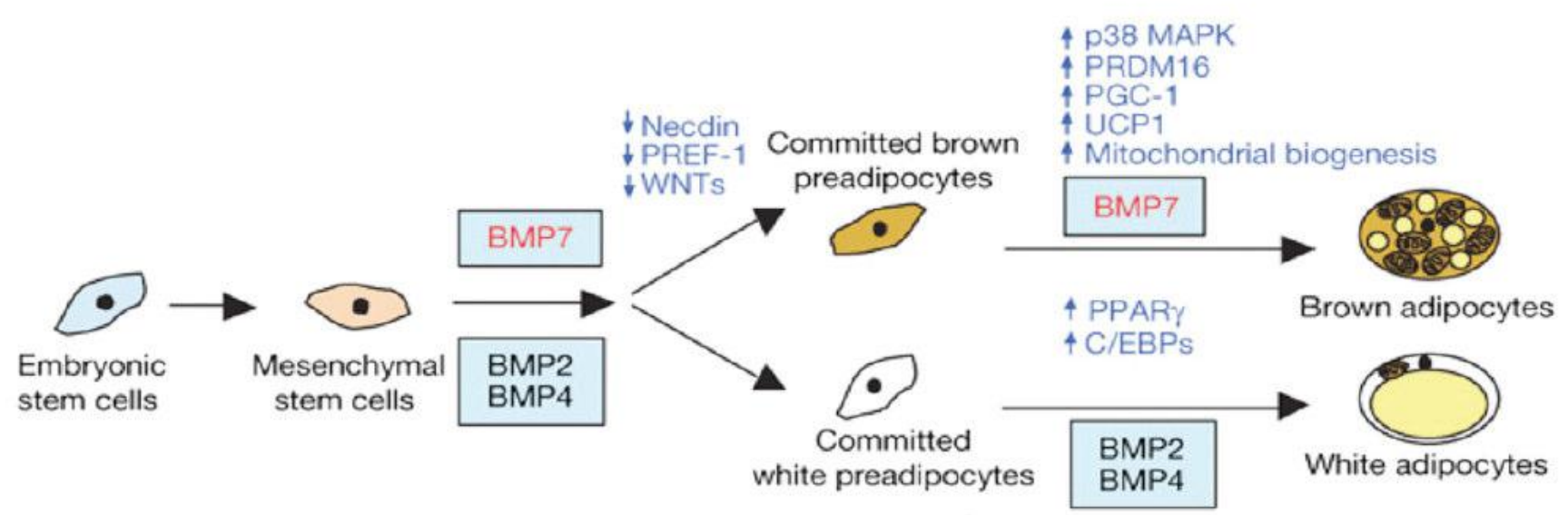

Figure 4. Evidence for an essential role of BMP-7 in BAT development and regulation of whole body energy expenditure by loss - of function and gain - of - function approaches (Adapted with permission from Nature Publishing Group) (40). 


\section{Human BAT: Antiobesity Therapeutic Visions}

As BAT is an adipose tissue in the sense that its cells accumulate lipid, the general concept has been that BAT produces heat by combusting this stored lipid. However, the stores are limited, and it has thus been recognized that lipids required for combustion are also obtained from the rest of the organism, particularly from chylomicrons in the circulation, through the activity of lipoprotein lipase. Additionally, brown fat mitochondria are equally well suited to carbohydrate combustion as to lipid combustion. The realization that BAT and muscle share a common origin (27) may make it easier to accept the concept that BAT is not a selective lipid consumer but will also readily combust carbohydrate (indeed it is glucose uptake that is seen in the PET scans) (44).

Thus, the glucose-utilizing activity of BAT could delay the development of a situation in which steadily increased glucose levels would successively lead to insulin resistance (44).

With the recognition that adult humans have in BAT an organ with substantial capacity to dissipate energy, targeting BAT thermogenesis may now be viewed as an appealing way to treat or prevent obesity and its associated disease. This is especially important because there are currently only three drugs approved by the FDA specifically for weight loss (sibutramine, phentermine, and orlistat), all of which focus primarily on the reduction on energy intake, and none of which have provided adequate long term clinical efficacy (45).

Looking forward to the future, there are two general approaches that could be used to increase BAT mass and activity: an in - vivo pharmacological approach using small molecules and growth factors (46-53) to stimulate BAT growth, differentiation, and activation, and an ex - vivo cell based approach, in which recent advances in brown adipogenesis could be used to increase brown fat differentiation from progenitor cells that in vitro could then be implanted in patients seeking weight loss.

The pharmacological approach is very attractive because increasing energy expenditure through thermogenesis has already proved to be very effective in achieving weight loss. Increasing energy expenditure may support the body's own adaptive response to weight gain. Interventions designed to increase BAT - mediated energy expenditure may be able to reset an obese individual's settling point for body weight back to a lower, healthier range (54).
Approaches to activate BAT by selective sympathetic activation have thus far had only limited success, early human trials using $\beta 3$ receptor agonists was not successful. This is at least in part, because human $\beta 3$ adrenergic receptors have different binding characteristics than those in rodents (55) and did not recognize some of the early ligands used in the clinical studies. Second generation $\beta 3$ agonists, which do bind human $\beta 3$ receptors, have poor oral bioavailability of unfavorable pharmacokinetics (47). One new generation $\beta 3$ agonists, L-796568, has been shown to acutely increase lipolysis and energy expenditure in overweight men (56), but its effect seemed to be lost after 28 days of treatments $(11,47)$.

A second approach for pharmacologic stimulation of BAT thermogenesis relies not on the traditional adrenergic pathways, but on newly discovered mechanisms involving BAT - specific growth factors (Fig. 4). For example, as noted earlier, the mesodermal growth factor BMP-7 can stimulate BAT growth and reduce weight gain when expressed systemically in mice (40).

The nexus between nutritional and pharmacological activators of BAT activity can be seen with the FGFs. Mice overexpressing FGF-21 are lean and have increased brown fat (58). FGF-21 can also be induced by a ketogenic diet (57) suggesting that some of the beneficial metabolic effects induced by a ketogenic diet are brought by increases in BAT mass. Through further details of his family's function remain to be determined, development of recombinant FGFs designed to stimulate BAT growth and activation hold promise as means for treating obesity (58).

In addition to these hormones, small - molecule regulators of proteins known to control brown adipogenesis, such as PGC- $1 \alpha, \mathrm{Rb}$, necdin, or PRDM16 may be developed. They could fill a niche similar to the thiasolidinedione, which may themselves improves insulin sensitivity through increased brown adipogenesis (59).

Although certainly in its earliest phases, recent studies (38) have suggested a new model for antiobesity treatment: ex - vivo manipulation to form functional BAT. The underlying principle that makes this an alluring option is that only gram - amounts of BAT are needed to have a meaningful metabolic impact (60). As noted earlier, at least two different routes of differentiation have been suggested to give rise to BAT (3), one leading to the major preformed depot of BAT in rodents, that is, the interscapular brown fat pad, which are derived from myf-5 expressing precursors (37) and the second involving systemic brown adipocytes that appear to be derived from myf- 5 negative precursors $(61,62)$.

Thus, in ex vivo approach, one would envision isolating progenitor cells from an individual by liposuction 
or muscle biopsy, then inducing brown adipogenesis using appropriate growth factors such as the BMPs, and transplanting them back into the original donor for invivo expansion. This methodology of autotransplantation could avoid unwarranted side effects of differentiating agents or growth factors and require only minimal surgical intervention. One challenge related to this novel methodology is whether increasing BAT mass will also result in increased activated BAT, which is required for thermogenesis and weight oss to occur (58).

\section{Thermogenic Ingredients}

The ultimate cause of obesity is an imbalance between energy intake and energy expenditure (EE). A negative energy balance is needed to produce weight loss and can be achieved by either decreasing intake or increasing expenditure.

Tools for obesity management, including consumption of caffeine, capsaicin and different teas such as green, white and oolong tea, have been proposed as strategies for weight loss and weight maintenance, as they may increase energy expenditure (4-5\%), fat oxidation (10-16\%) and have been proposed to counteract the decrease in metabolic rate that is present during weight loss. Daily increases in thermogenesis of approximately $300-400 \mathrm{~kJ}$ can eventually lead to substantial weight loss (63).

Catechins in tea inhibit the enzyme catechol O-methyltransferase (COMT) that is present in almost every tissue and degrades catecholic compounds such as norepinephrine (NE) $(64,65)$. COMT decreases the hydrophilicity by methylation, followed by sulfation and glucuronidation to make the excretion in urine and bile possible (66). NE cannot be degraded through the inhibition of COMT, and consequently the sympathetic nerve system (SNS) will be stimulated continuously due to the presence of NE, which attaches to b-adrenoceptors and causes an increase in $\mathrm{EE}$ and fat oxidation.

As caffeine is also present in tea, its effect will also take place after tea consumption. Caffeine affects the thermogenesis by inhibiting the enzyme phosphodiesterase. This enzyme degrades intracellular cyclic amino mono phosphate (67). Phosphodiesterase usually hydrolyses cyclic adenosine monophosphate (cAMP) to AMP, but after consumption of caffeine, cAMP concentration rises and SNS activity will be increased and inactive hormonesensitive lipase will be activated, which promotes lipolysis (68). The SNS activity and lipoysis are dependent on cAMP, because cAMP activates the protein kinase A (69). Besides the inhibition of phosphodiesterase, caffeine also affects the thermogenesis through the stimulation of substrate cycles such as the Cori-cycle and the FFA-triglyceride cycle.

Capsaicin has been reported to increase thermogenesis by enhancing catecholamine secretion from the adrenal medulla in rats, mainly through activation of the central nervous system. Increase in thermogenesis induced by capsaicin is probably based on $\beta$-adrenergic stimulation. Both animal and human studies showed that the increase in thermogenesis is abolished after administration of $\beta$-adrenergic blockers such as propranolol. The upregulation of UCPs 1 and 2 after capsiate administration in animals was showed by Masuda et al (70) and believed to be responsible for the increase in thermogenesis.

Ingredients for obesity management are tea, caffeine and capsaicin, as they increase daily EE with 4-5\% (.300$400 \mathrm{~kJ}$ ) without increasing energy intake and counteract the decrease in metabolic rate during weight loss. Studies have shown that these ingredients are useful in losing weight or preventing weight regain after weight loss (63).

\section{Conclusions}

The obesity pandemic requires new and novel treatments. The past few years have witnessed multiple studies conclusively showing that adult humans have functional BAT, a tissue that has a tremendous capacity for obesity - reducing thermogenesis. This leads to a paradigm shift in the understanding of human metabolism and of obesity. Adaptive adrenergic thermogenesis in humans represents BAT activity, the absence of which may contribute to obesity. Although many questions remain regarding efficacy, safety, practically, and durability of such treatments, we are encourage that novel therapies targeting BAT thermogenesis may be available in the near future as therapies for obesity and diabetes.

\section{References:}

1. Bellisari A. Evolutionary origins of obesity. Obes Rev 2008; 9 : 165-180.

2. Celi FS. Brown adipose tissue - when it pays to be inefficient. N Engl J Med 2009; 360: 1553 - 1556.

3. Gesta S, Tseng YH, Kahn CR. Developmental origin of fat: tracking obesity to its source. Cell 2007; 131: $242-256$.

4. Cannon B, Nedergaard J. Brown adipose tissue: function and physiological significance. Physiol Rev 2004; 84: $277-$ 359. 
5. Silva JE, Larsen PR. Adrenergic activation of triiodothyronine production in brown adipose tissue. Nature 1983; 305 . $712-713$

6. Watanabe $\mathrm{M}$, Houten SM, Mataki $\mathrm{C}$, et al. Bile acids induce energy expenditure by promoting intracellular thyroid hormone activation. Nature 2006; 439: 484-489.

7. Crisan M, Casteilla L, Lehr L, et al. A reservoir of brown adipocyte progenitors in human skeletal muscle. Stem Cells 2008; 26: 2425 - 2433.

8. Heaton JM. The distribution of brown adipose tissue in the human. J Anat 1972; 112: $35-39$.

9. Astrup A. Thermogenesis in human brown adipose tissue and skeletal muscle induced by sympathomimetic stimulation. Acta Endocrinol Suppl (Copenh) 1986; 278: 1 - 32.

10. Weyer C, Tataranni PA, Snitker S, et al. Increase in insulin action and fat oxidation after treatment with $\mathrm{CL} 316,243$, a highly - selective beta3-adrenoceptor agonist in humans. Diabetes 1998; 47: 1555 - 1561.

11. Larsen TM, Toubro S, van Baak MA, et al. Effect of 28-d treatment with L-796568, a novel beta(3)-adrenergic receptor agonist, on energy expenditure and body composition in obese men. Am J Clin Nutr 2002; 76: 780 -788 .

12. Nedergaard J, Bengtsson T, Cannon B. Unexpected evidence for active brown adipose tissue in adult humans. Am J Physiol Endocrinol Metab 2007; 293: E444 - E452.

13. Cunningham $S$, Leslie $P$, Hopwood $D$, et al. The characterization and energetic potential of brown adipose tissue in man. Clin Sci (Lond) 1985; 69: $343-348$.

14. Schoder $H$, Larson SM, Yeung HW. PET/CT in oncology integration into clinical management of lymphoma, melanoma, and gastrointestinal malignancies. J Nucl Med 2004; 45 (Suppl 1: 72S - 81S

15. Hany TF, Gharehpapagh E, Kamel EM, et al. Brown adipose tissue: a factor to consider in symmetrical tracer uptake in the neck and upper chest region. Eur $\mathrm{J}$ Nucl Med Mol Imaging 2002; 29: 1393 - 1398

16. Cohade C, Osman M, Pannu HK, Wahl RL. Uptake in supraclavicular area fat ("USA fat"): description on $18 \mathrm{~F}$ FDG PET/CT. J Nucl Med 2003; 44: 170 - 176.

17. van Marken Lichtenbelt WD, Vanhommerig JW, Smulders NM, et al. Cold-activated brown adipose tissue in healthy men. N Engl J Med 2009; 360: 1500-1508.

18. Saito M, Okamatsu - Ogura Y, Matsushita M. High incidence of metabolically active brown adipose tissue in healthy adult humans. Effects of cold exposure and adiposity. Diabetes 2009; 58: 1526 - 1531

19. Enerbäck S. Human brown adipose tissue. Cell Metab 2010; 11: $248-252$.

20. Cannon B, Nedergaard, J. Brown adipose tissue: function and physiological significance. Physiol Rev 2004; 84: 277359

21. Frontini A, Cinti S. Distribution and development of brown adipocytes in the murine and human adipose organ. Cell Metab 2010; 11: $253-256$.

22. Bachman ES, Dhillon $\mathrm{H}$, Zhang $\mathrm{CY}$, et al. betaAR signaling required for diet-induced thermogenesis and obesity resistance. Science 2002; 297: 843-845

23. Kopecky J, Clarke G, Enerbäck S, Spiegelman B, Kozak LP. Expression of the mitochondrial uncoupling protein gene from the aP2 gene promoter prevents genetic obesity. J Clin Invest 1995; 96: 2914-2923.

24. Kozak LP. Brown fat and the myth of diet - induced thermogenesis. Cell Metab 2010; 11: $263-267$.
25. Foster DO, Frydman ML. Nonshivering thermogenesis in the rat. II. Measurements of blood flow with microspheres point to brown adipose tissue as the dominant site of the calorigenesis induced by noradrenaline. Can J Physiol Pharmacol. 1978; 56: 110-122

26. Farmer SR. Transcriptional control of adipocyte formation. Cell Metab 2006; 4: 263 - 273.

27. Kajimura S, Seale P, Spiegelman BM. Transcriptional control of brown fat development. Cell Metab 2010; 11: $257-262$.

28. Cederberg A, Grønning LM, Ahrén B, Taskén K, Carlsson $\mathrm{P}$, Enerbäck $\mathrm{S}$. FOXC2 is a winged helix gene that counteracts obesity, hypertriglyceridemia, and dietinduced insulin resistance. Cell 2001; 106: 563 - 573.

29. Puigserver P, Wu Z, Park CW, Graves R, Wright M, Spiegelman BM. A cold-inducible coactivator of nuclear receptors linked to adaptive thermogenesis. Cell 1998; 92: 829 839.

30. Leonardsson G, Steel JH, Christian M, et al. Nuclear receptor corepressor RIP140 regulates fat accumulation. Proc Natl Acad Sci U S A 2004; 101: 8437-8442.

31. Picard F, Géhin M, Annicotte J, et al. SRC-1 and TIF2 control energy balance between white and brown adipose tissues. Cell 2002; 111: 931-941.

32. Hansen JB, Jørgensen C, Petersen RK, et al. Retinoblastoma protein functions as a molecular switch determining white versus brown adipocyte differentiation. Proc Natl Acad Sci U S A. 2004; 101: 4112-4117.

33. Scimè A, Grenier G, Huh MS, et al. Rb and p107 regulate preadipocyte differentiation into white versus brown fat through repression of PGC-1alpha. Cell Metab 2005; 2 : 283-295.

34. Pan D, Fujimoto M, Lopes A, Wang YX. Twist-1 is a PPARdeltainducible, negative-feedback regulator of PGC-1alpha in brown fat metabolism. Cell 2009; 137: $73-86$.

35. Mochizuki N, Shimizu S, Nagasawa T, et al. A novel gene, MEL1, mapped to $1 \mathrm{p} 36.3$ is highly homologous to the MDS1/EVI1 gene and is transcriptionally activated in $\mathrm{t}(1 ; 3)(\mathrm{p} 36 ; \mathrm{q} 21)$-positive leukemia cells. Blood. 2000; 96: 3209-3214

36. Seale P, Kajimura S, Yang W, et al. Transcriptional control of brown fat determination by PRDM16. Cell Metab. 2007; 6 : 38-54.

37. Seale $\mathrm{P}$, Bjork B, Yang W, et al. PRDM16 controls a brown fat/ skeletal muscle switch. Nature. 2008; 454: 961-967.

38. Kajimura S, Seale P, Kubota K, et al. RInitiation of myoblast to brown fat switch by a PRDM16-C/EBP-beta transcriptional complex. Nature. 2009; 460: 1154-1158.

39. Karamitri A, Shore AM, Docherty K, Speakman JR, Lomax MA. Combinatorial transcription factor regulation of the cyclic AMP-response element on the Pgc-1alpha promoter in white 3T3-L1 and brown HIB-1B preadipocytes. J Biol Chem. 2009; 284: 20738-20752.

40. Tseng YH, Kokkotou E, Schulz TJ, et al. New role of bone morphogenetic protein 7 in brown adipogenesis and energy expenditure. Nature. 2008; 454: 1000-1004.

41. Shen JJ, Huang L, Li L, Jorgez C, Matzuk MM, Brown CW. Deficiency of growth differentiation factor 3 protects against diet-induced obesity by selectively acting on white adipose. Mol Endocrinol. 2009; 23: 113-123.

42. Ross SE, Hemati N, Longo KA, et al. Inhibition of adipogenesis by Wnt signaling. Science. 2000; 28: 950-953.

43. Konishi M, Mikami T, Yamasaki M, Miyake A, Itoh N. Fibroblast growth factor-16 is a growth factor for embryonic brown adipocytes. J Biol Chem. 2000; 275: 12119-12122. 
44. Nedergaard J, Cannon B. The changed metabolic world with human brown adipose tissue: therapeutic visions. Cell Metab 2010; 11: 268 - 272.

45. Melnikova I, Wages D. Antiobesity therapies. Nat Rev Drug Discov 2006; 5: 369 - 370 .

46. Shekelle PG, Hardy ML, Morton SC, et al. Efficacy and safety of ephedra and ephedrine for weight loss and athletic performance: a meta - analysis. JAMA 2003; 289: 1537 -1545 .

47. Arch JR. The discovery of drugs for obesity, the metabolic effects of leptin and variable receptor pharmacology: perspective from beta3-adrenoreceptor agonists. Naunyn Schmiedebergs Arch Pharmacol 2008; 378: 225 - 240.

48. Tiwari A, Maiti P. TGR5: an emerging bile acid G - protein - coupled receptor target for the potential treatment of metabolic disorders. Drug Discov Today 2009; 14: 523 530 .

49. Thomas C, Gioielio A, Noriega L, et al. TGR5 - mediated bile acid sensing controls glucose homeostasis. Cell Metab 2009; 10: $167-177$.

50. Kendall DM, Rubin CJ, Mohideen P, et al. improvement of glycemic control, triglycerides, and HDL cholesterol levels with muraglitazar, a dual (alpha/gamma) peroxisome proliferator - activated receptor activator, in patients with type 2 diabetes inadequately controlled with metformin monotherapy: a double - blind, randomized, pioglitazone - comparative study. Diab Care 2006; 29: 1016 - 1023.

51. Henry RR, Lincoff $A M$, Mudaliar $S<$ et al. Effect of the dual peroxisome proliferator - activated receptor - alpha/ gamma agonist aleglitazar on risk of cardiovascular disease in patients with type 2 diabetes (SYNCHRONY): a phase II, randomized, dose - ranging study. Lancet 2009; 374: $126-136$

52. Yamamoto $\mathrm{H}$, Schoonjans $\mathrm{K}$, Auwerx J. Sirtuin functions in health and disease. Mol Endocrinol 2007; 21: 1745 1755 .

53. Wang $\mathrm{YX}$, Lee $\mathrm{CH}$, Tiep $\mathrm{S}$, et al. Peroxisome proliferator - activated receptor delta activates fat metabolism to prevent obesity. Cell 2003; 113: $159-170$.

54. Harper ME, Green K, Brand MD. The efficiency of cellular energy transduction and its implications for obesity. Annu Rev Nutr 2008; 28: 13 - 33

55. Clapham JC, Arch JR. Thermogenic and metabolic antiobesity drugs: rationale and opportunities. Diabetes Obes Metab 2007; 9: 259 - 275.

56. Van Baak MA, Hui GB, Toubro $\mathrm{S}<$ et al. Acute effect of L-796568, a novel beta 3-adrenergic receptor agonist, on energy expenditure in obese men. Clin Pharmacol Ther 2002; 71: 272 - 279 .

57. Badman MK, Pissios P, Kennedy AR, et al. Hepatic fibroblast growth factor 21 is regulated by PPARaplha and is a key mediator of hepatic lipid metabolism in ketotic states. Cell Metab 2007; 5: 426 - 437.
58. Cypess AM, Kahn CR. Brown fat as a therapy for obesity and diabetes. Curr Opin Endocrinol 2010; 17: 143 - 149.

59. Vernochet C, Peres SB, Davis KE, et al. C/EBPalpha and the corepresors CtBP1 and CtBP2 regulate repression of select visceral white adipose genes during induction of the brown phenotype in white adipocytes by peroxisome proliferator - activated receptor gamma agonists. Mol Cell Biol 2009; 29: 4714 - 4728 .

60. Rothwell NJ, Stock MJ, Luxuskonsumption, diet - induced thermogenesis and brown fat: the case in favour. Clin Sci (Lond) 1983; 64: 19 - 23.

61. Almind K, Manieri M, Sivitz WI, et al. Ectopic brown adipose tissue in muscle provides a mechanisms for differences in risk of metabolic syndrome in mice. Proc Natl Acad Sci U S A 2007; 104: 2366 - 2371.

62. Xue B, Rim JS, Hogan CJ, et al. genetic variability affects the development of brown adipocytes in white fat but not in interscapular brown fat. J Lipid Res 2007; 48: 41 - 51.

63. HurselR, Westerterp-Plantenga MS. Thermogenic ingredients and body weight regulation. Int J Obes 2010; 34: 659 669.

64. Dulloo AG, Duret C, Rohrer D, et al. Efficacy of a green tea extract rich in catechin polyphenols and caffeine in increasing 24-h energy expenditure and fat oxidation in humans. Am J Clin Nutr 1999; 70: 1040-1045.

65. Westerterp-Plantenga MS, Lejeune MP, Kovacs EM. Body weight loss and weight maintenance in relation to habitual caffeine intake and green tea supplementation. Obes Res 2005; 13: 1195-1204.

66. Shixian Q, VanCrey B, Shi J, Kakuda Y, Jiang Y. Green tea extract thermogenesis-induced weight loss by epigallocatechin gallate inhibition of catechol-O-methyltransferase. J Med Food 2006; 9: 451-458.

67. Cornelis MC, El-Sohemy A, Campos H. Genetic polymorphism of the adenosine A2A receptor is associated with habitual caffeine consumption. Am J Clin Nutr 2007; 86: 240-244.

68. Acheson KJ, Gremaud G, Meirim I, et al. Metabolic effects of caffeine in humans: lipid oxidation or futile cycling? Am J Clin Nutr 2004; 79: 40-46.

69. Belza A, Frandsen E, Kondrup J. Body fat loss achieved by stimulation of thermogenesis by a combination of bioactive food ingredients: a placebo-controlled, doubleblind 8-week intervention in obese subjects. Int $\mathrm{J}$ Obes 2007; 31: 121-130.

70. Masuda Y, Haramizu S, Oki K, et al. Upregulation of uncoupling proteins by oral administration of capsiate, a nonpungent capsaicin analog. J Appl Physiol 2003; 95: 2408-2415. 